\title{
DroidArts - Aplicativo para a Visualização e Interação com Imagens em Aulas de Artes
}

\author{
Luiz Carlos Pereira Filho ${ }^{1}$, Luciano Bernardes de Paula ${ }^{1}$ \\ ${ }^{1}$ Instituto Federal de Educação, Ciência e Tecnologia de São Paulo (IFSP) \\ Câmpus de Bragança Paulista \\ Av. Francisco Samuel Lucchesi Filho, 770, Penha, Bragança Paulista - SP \\ Brasil - CEP: 12929-600 \\ luizcarlos.pereirafilho@yahoo.com.br, lbernardesdifsp.edu.br
}

\begin{abstract}
In this paper the development of a app for mobile devices using Android for Arts classes is presented. The app is used to view and to interact with images and pictures, allowing teachers to plan activities about an image (a painting, for example), adding notes, and the students, starting from the teacher's notes, may create their own notes. These notes from the students are submitted to a cloud system, allowing the teacher to posteriorly evaluate them. Real tests were done with students of Art classes of a public educational institution and the results are presented in this paper.
\end{abstract}

Resumo. Este artigo apresenta o desenvolvimento de um aplicativo para dispositivos móveis utilizando Android para uso em aulas de Artes. O aplicativo é utilizado para visualização e interação com imagens e figuras, permitindo que professores planejem atividades a respeito de uma imagem (uma pintura, por exemplo), adicionando anotações e os alunos, a partir da anotações feitas pelo docente, criem suas próprias. As anotações dos alunos são submetidas para um sistema em nuvem, permitindo que o docente avalie posteriormente. Foram feitos testes reais com alunos da disciplina de artes de uma instituição pública de ensino e os resultados são apresentados neste artigo.

\section{Introdução}

O contexto principal deste trabalho gira em torno da colaboração e cooperação com o processo de ensino tradicional e consolidado em salas de aula do ensino médio por meio da incorporação de Tecnologias da Informação e Comunicação (TIC). São evidentes as transformações causadas pelas TIC em diversos setores da vida e áreas dos saberes. Exemplos simples são as telecomunicações, a imprensa e a medicina, que se apoiaram na evolução das TIC para elevar a qualidade dos serviços prestados, além da aquisição, preservação e proliferação de conhecimento de forma trivial [Ferrete and Ferrrete 2015].

A aplicação das TIC em ambiente de ensino demanda algumas mudanças tanto físicas quanto institucionais. Além disso, mudanças comportamentais por partes de alunos e professores são necessárias. A apropriação tecnológica é intrínseca da incorporação, tanto por parte de discentes quanto por parte de docentes.

Segundo [Ramos 2011], para a efetividade do ensino, é necessária a contextualização do conteúdo abordado com o método didático usado, tanto pela teoria quanto pela prática. Aplicações descontextualizadas podem levar a resultados que 
VI Congresso Brasileiro de Informática na Educação (CBIE 2017)

Anais dos Workshops do VI Congresso Brasileiro de Informática na Educação (WCBIE 2017)

são impeditivos para a continuidade da tentativa de implantação de métodos diferentes dos tradicionais. No contexto atual, a tecnologia mobile facilita o acesso à Internet e a aplicativos com diversos propósitos, dando conectividade a qualquer um, em qualquer ambiente, com comodidade e simplicidade [Mendonça 2015].

O objetivo geral deste trabalho, desenvolvido como um trabalho de conclusão de curso, foi criar um aplicativo para dispositivos móveis com foco na interação com imagens para aplicação em salas de aula da disciplina de artes do ensino médio, possibilitando a contextualização da tecnologia com o ambiente de ensino e o conteúdo aplicado, viabilizando a aplicação em atividades interativas.

A disciplina de artes foi escolhida para a demonstração do objetivo proposto, uma vez que trata-se de uma área do conhecimento na qual o uso de imagens (quadros, fotografias, gravuras) é de suma importância. Outro fator motivador foi a existência de sites de museus internacionais que disponibilizam imagens digitais em grande resolução de seu acervo, o que possibilita o uso destas no contexto da disicplina.

\section{As Tecnologias da Informação e Comunicação na Educação}

As TIC trouxeram grande impacto na sociedade atual, tanto no uso individual quanto no coletivo, e em todas as classes econômicas. No atual contexto de transformações tecnológicas práticas inovadoras devem ser aplicadas, levando a legitimidade da representação do conhecimento, dando alternativas que possam levar a uma formação completa do aluno para a vida em sociedade [Pedro et al. 2012]. [Pretto 2007] destaca quatro fases para apropriação das TIC na educação baseada nos estudos desenvolvidos por profissionais em educação e informática.

Em uma primeira fase, Papert e Piaget influenciaram o uso de computadores em um tempo que os mesmos apresentavam capacidade reduzida de processamento e armazenamento. A linguagem de programação Logo era utilizada com âmbito no universo lógico-formal. Em um segundo momento, entra em cena a fase dos computadores digitais e em rede, na qual os governos passaram a equipar escolas com computadores. Os portais educacionais fazem parte da terceira fase, onde conteúdos e informações são centralizados, podendo ser disponibilizados em massa. Na fase atual, a Web 2.0 tornou os recursos de navegação pela rede mais simples e intuitivos, abrindo possibilidades de uso, como o compartilhamento de conteúdo com outros usuários [Pretto 2007].

Com os recursos computacionais inovadores, novas e eficientes tecnologias e as crescentes pesquisas na área educacional contribuem para a reestruturação do modo clássico e tradicional de ensinar e aprender. Os recursos tecnológicos disponíveis podem colaborar para a reforma e inovação da educação. A incorporação de novas tecnologias traz dinamismo e vinculações entre contextos de ensino e culturas que se desenvolvem fora do âmbito escolar.As aptidões da atual geração com as novas tecnologias contribuem muito para o sucesso da renovação dos métodos de ensino e aprendizagem [Pedro et al. 2012].

[Silva 2001] pontua que software ou aplicativos, quando aplicado ao ambiente de ensino e aprendizagem, devem motivar o aluno a obter conhecimento e envolvê-lo na construção de seu próprio conhecimento.

Para [Mercado 2002], esse novo paradigma de educação exige uma reflexão sis- 
VI Congresso Brasileiro de Informática na Educação (CBIE 2017)

Anais dos Workshops do VI Congresso Brasileiro de Informática na Educação (WCBIE 2017)

temática acerca de seus objetivos, pois mudam as formas de aprender e as competências exigidas para um educador. A formação de um educador se torna algo contínuo, pois terá a tecnologia mediando o processo de ensino e aprendizagem.

\subsection{Recursos Visuais e Imagens na Educação}

Segundo [Rodrigues 2007], imagens são definidas como representações visuais, podendo representar diversos objetos, seres ou conceitos, além de possuir representação concreta e abstrata. A representação concreta remete a manifestações físicas, enquanto a representação abstrata é relacionada a imagens criadas mentalmente pelos indivíduos.

As imagens oriundas da comunicação são categorizadas em artesanais e técnicas. As imagens artesanais são produzidas com o uso de instrumentos, como pincel, lápis e instrumentos de percussão, por exemplo. Essas são as imagens tradicionais por serem oriundas dos primórdios da humanidade. Pinturas, desenhos e esculturas são integrantes desse grupo de imagens [Costa 2013].

Com os avanços tecnológicos do século XX, os níveis de compreensão da imagem se elevaram, viabilizando sua aplicação fora do meio da comunicação. As imagens passaram a apoiar, mais significativa e efetivamente, atividades relacionadas à pesquisa e ao ensino, tornando-se objeto de informação e conhecimento [Rodrigues 2007].

As vantagens em se aplicar imagens e recursos visuais em um ambiente de ensino estão no despertar de reações e emoções que isso pode provocar, impactando positivamente o observador. Um aspecto vantajoso da aplicação de imagens para educar é o fato da evolução tecnológica disponibilizar um número significativo de imagens de diversas origens com diversos fins, citando como exemplo pinturas e desenhos, imagens de cinemas, televisão e computador. As imagens passaram de simples complemento ao processo de ensino e aprendizagem, a um importante componente da produção científica [Costa 2013].

Todos esses aspectos são ainda mais potencializados no contexto da área de conhecimento conhecida como Artes, na qual imagens, pinturas, entre outras formas de expressão possuem o papel de transmitir emoções e registrar o passado.

Desta forma, este trabalho tem o objetivo de desenvolver e testar um aplicativo para dispositivos móveis utilizando Android para auxiliar docentes e alunos em aulas de Artes. O principal objetivo do aplicativo é fornecer uma plataforma interativa para que o docente possa criar e organizar aulas sobre determinada imagem, figura ou pintura. Uma vez definida a imagem a ser utilizada na aula, o docente pode criar anotações a respeito da mesma. No momento da aula, os alunos poderão, utilizando o mesmo aplicativo, percorrer a imagem de forma livre, utilizando gestos para deslizar pela imagem e também recursos de zoom. Os alunos podem encontrar as anotações feitas pelo docente e até mesmo criar suas próprias anotações.

O aplicativo está vinculado a um servidor na nuvem que recebe todas as anotações feitas pelos alunos, possibilitando que o docente, posteriormente, verifique as mesmas, podendo avaliar a compreensão dos discentes a respeito da atividade proposta. 
VI Congresso Brasileiro de Informática na Educação (CBIE 2017)

Anais dos Workshops do VI Congresso Brasileiro de Informática na Educação (WCBIE 2017)

\section{Trabalhos relacionados}

$\mathrm{Na}$ literatura encontram-se algumas ferramentas desenvolvidas para o ensino via dispositivos móveis. Em [Melo and Carvalho 2014] é exposto que a aprendizagem móvel cria uma rede ampla de comunicação e de oportunidades, aumentando as possibilidades de ensino com o reposicionamento da sala de aula e todos os espaços fora dela como lugares para ensinar e aprender. O estudo dos autores destaca a importância do ensino e aprendizagem via dispositivos móveis. Destacam-se a facilidade de acesso à aparelhos móveis e a ampliação das oportunidades de aprendizagem, ampliando o acesso à conteúdos pedagógicos, criação de comunidades de aprendizagem ativa, interativa e colaborativa. A seguir serão apresentados alguns trabalhos de relevância para o tema proposto.

\subsection{Geometria Interativa}

O trabalho de [Pedro et al. 2012] apresenta estudo, planejamento e desenvolvimento de uma ferramenta de Geometria Interativa (GI), ou Geometria Dinâmica(GD), para ser utilizada em dispositivos móveis baseados na tecnologia Android. Foi identificado um modelo adequado de interface e interação para a ferramenta, de modo a se obter um ambiente de aprendizagem alinhado aos princípios de GI.

Desenvolvido na plataforma Android, o aplicativo realiza funcionalidades básicas de um software de GI, como: criação, remoção e manipulação de pontos, retas e circunferências; a criação de interseções entre retas e circunferências; a criação de pontos pertencentes a uma reta ou a uma circunferência e criação de interseções entre retas e circunferências.

\subsection{GeoTouch}

O aplicativo GeoTouch é um aplicativo criado para auxiliar no ensino e aprendizagem de geometria em salas de aula. [Reis 2014] desenvolveu um trabalho investigativo em torno de manipulação dos eventos de toque do Android para auxiliar o desenvolvimento de aplicativos de interação didática. O objetivo do trabalho foi desenvolver um conjunto de gestos para a construção de objetos geométricos em um aplicativo para ensinar geometria em dispositivos móveis com a tela sensível ao toque.

\subsection{Carbópolis}

O Carbópolis foi desenvolvido pela área de educação Química do Instituto de Química da Universidade Federal do Rio Grande do Sul (UFRGS), juntamente com o Programa Especial de Treinamento do Instituto de Informática da própria UFRGS. Foi desenvolvido com a linguagem Java e com o banco de dados Mysql .

[Eicher and Pino 2015], os criadores de Carbópolis relatam que o aplicativo possui um ambiente para debate de questões ambientais e foi desenvolvido para alunos e professores de diferentes níveis de ensino. Foram criados cenários que constituem a cidade Carbópolis com várias situações.

A pesquisa a respeito dos trabalhos relacionados mostrou proveitosa, indicou a grande gama de aplicativos e software voltados para educação, porém não foi encontrado nenhum específico para Artes, o que configura um diferencial do presente trabalho. 
VI Congresso Brasileiro de Informática na Educação (CBIE 2017)

Anais dos Workshops do VI Congresso Brasileiro de Informática na Educação (WCBIE 2017)

\section{Desenvolvimento e Tecnologias}

Segundo [Lee 2011], uma grande parcela dos smartphones e tablets presentes no mercado são compatíveis com o Android e, baseando-se nisso, foram usados no projeto para testes, validações e ensaios, bem como para a aplicação no ambiente real do problema.

Linguagem de programação, bibliotecas e APIs do Java foram usadas por serem compatíveis com o Android. O Android Studio foi a IDE utilizada, tendo sido escolhida por ser considerada pela Google como a ferramenta oficial para desenvolvimento Android, permitindo a codificação, compilação, depuração e emulação de aplicativos ${ }^{1}$.

Foram estudadas APIs para manipulação de eventos de toques diversos e gestos para verificação de viabilidade de aplicação. Os métodos manipuladores de gestos e toques existentes no Android foram aplicados, pois existe a interação com imagens e com o controle de zoom e foco durante quase todo o tempo de trabalho com o aplicativo no ambiente de ensino.

Para validações, testes e demonstrações foi desenvolvido um protótipo para tablets e smartphones, que torna a aplicação responsiva quanto a sua interface gráfica.

\subsection{Aplicativo desenvolvido}

A Figura 1 ilustra a arquitetura do sistema, que possui, além do aplicativo (denominado de DroidArts na figura), a representação do sistema cliente/servidor, explorado mais a diante nesta subseção, e a representação de um repositório de museu, fonte das obras de artes utilizadas no aplicativo (representado na figura pelo símbolo do The Metropolitan Museum of $\mathrm{Art}^{2}$, um dos museus internacionais que disponibilizam seu acervo de forma digital). Os dados entre o aplicativo e sistema cliente-servidor trafegam usando o padrão JavaScript Object Notation (JSON - Notação de Objeto JavaScript). As imagens do repositório do museu são obtidas por meio requisições HTTP (Hipertext Transfer Protocol).

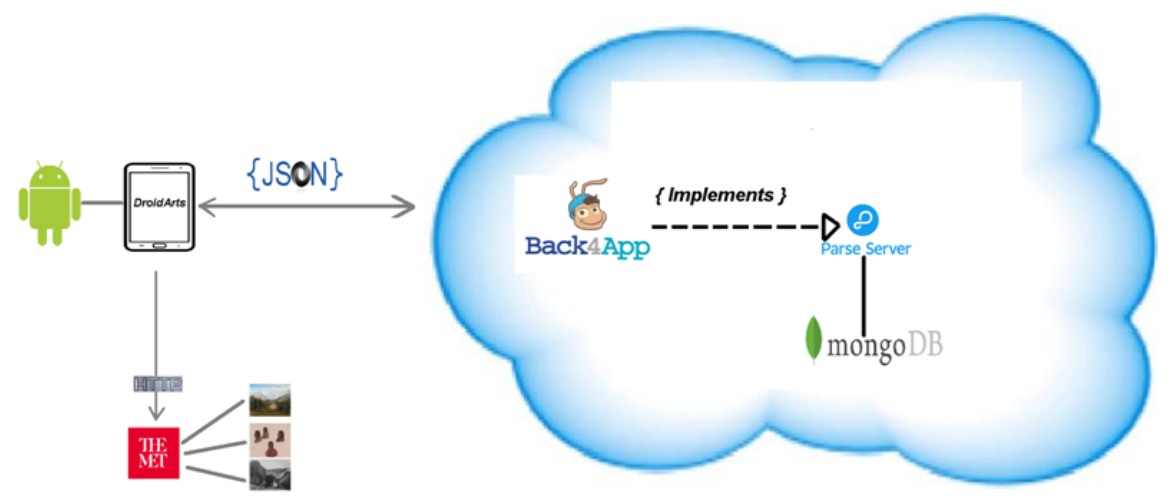

Figura 1. Arquitetura do sistema.

Como backend foi utilizado o Back4app ${ }^{3}$, um serviço de processamento e armazenamento disponível na Internet. O Back4app é um BaaS (backend as a service) e oferece,

\footnotetext{
${ }^{1}$ Android Developer - http://developer.android.com - acessado em 20 de agosto de 2017

${ }^{2}$ The Metropolitan Museum of Art de Nova York - http://www.metmuseum.org/ - acessado em 20 de agosto de 2017

${ }^{3}$ Back4app - http://back4app.com - acessado em 20 de agosto de 2017
} 
VI Congresso Brasileiro de Informática na Educação (CBIE 2017)

Anais dos Workshops do VI Congresso Brasileiro de Informática na Educação (WCBIE 2017)

de forma simples, hospedagem de backend para aplicativos móveis. O Back4app utiliza o ParseServer que, por sua vez, utiliza o MongoDB, um banco de dados NoSQL.

A API do Android disponibiliza recursos relacionados a eventos de interação com o usuário, e isso foi explorado para o desenvolvimento de funcionalidades de zoom, foco, visualização de informações na tela relacionadas às imagens. Além desses recursos da API, foi utilizada a biblioteca Picasso, usada para executar tarefas de downloads e trabalhar com bitmaps de arquivos de imagens.

Um dos objetivos específicos deste projeto era testar o aplicativo no ambiente real do problema. Desta forma, foram realizados testes com turmas da disciplina de artes do ensino médio de uma instituição de ensino pública.

\subsection{Descrição do aplicativo}

Inicialmente, o aluno deve se cadastrar no sistema e, após isso, há uma tela em que o aluno poderá visualizar as tarefas ativas cadastradas pelo professor. Da mesma forma, o professor poderá visualizar as tarefas ativas e cadastradas por ele/ela anteriormente é semelhante a tela de visualização do aluno.

Caso o aluno selecione uma das tarefas, ele será redirecionado para uma tela onde poderá realizar a tarefa e enviá-la para avaliação. No caso do professor, ao selecionar uma tarefa, será mostrado na tela os alunos que realizaram e enviaram a mesma para avaliação.

Para criar uma nova tarefa, são necessários alguns passos até a disponibilização para os alunos. O professor deve inserir o link para o repositório de um museu e apertar o botão que faz o download da imagem.

A Figura 2 ilustra a imagem de uma obra de arte obtida do repositório do Museu Metropolitano de Arte de Nova York. Após o download da imagem da obra de arte escolhida, o professor poderá interagir com a mesma, manipulando, de forma dinâmica, zoom e foco e inserindo e visualizando comentários, observações e perguntas em qualquer ponto da obra de arte.
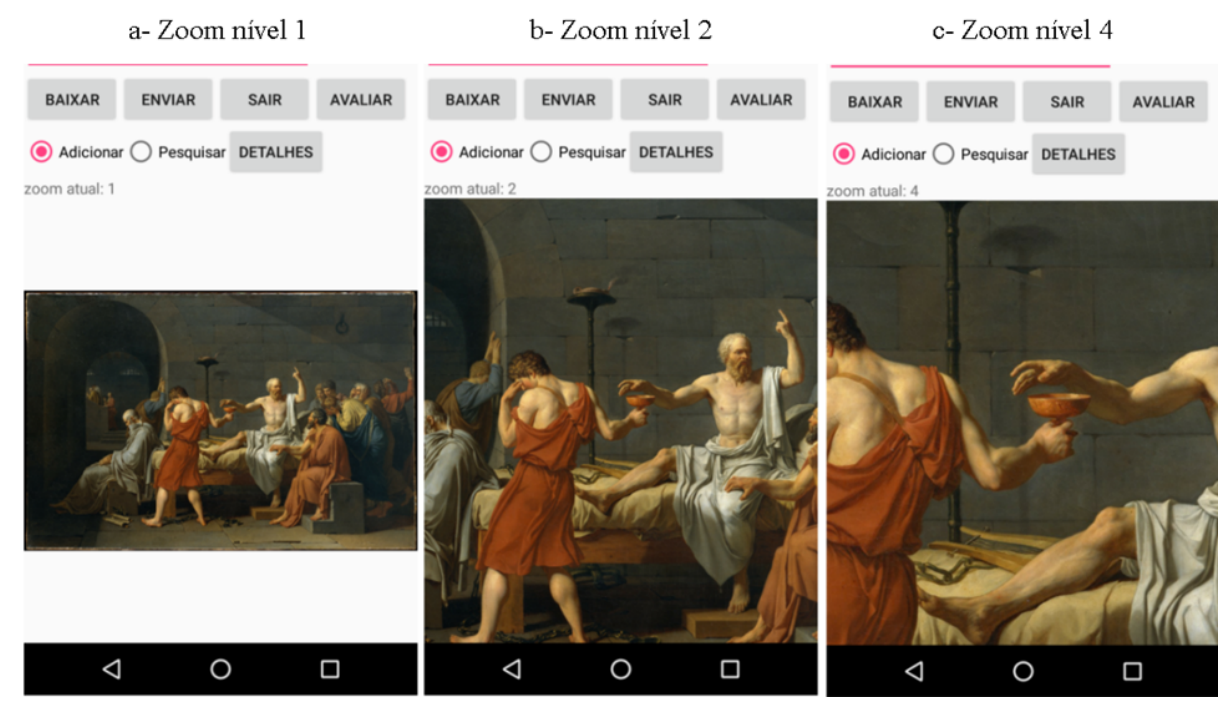

Figura 2. Zoom aplicado à imagem. Obra intitulada "The Death of Socrates"[David 1787] 
VI Congresso Brasileiro de Informática na Educação (CBIE 2017)

Anais dos Workshops do VI Congresso Brasileiro de Informática na Educação (WCBIE 2017)

A interação por meio do ajuste do zoom é ilustrada nas Figuras 2-a, 2-b e 2-c. O usuário tem a liberdade para aumentar ou diminuir o zoom até o nível em que desejar. A tela conta com um campo que indica dinamicamente, o nível de zoom atual.

Além da manipulação de zoom e foco, o professor conta com a funcionalidade de adição de comentários, observações e perguntas. Com a opção Adicionar marcada, basta dar um clique em qualquer ponto da imagem para receber uma caixa para inserção de texto. A quantidade de comentários e caracteres que podem ser inseridos é ilimitada, possibilitando a criação de uma tarefa detalhada.

Para visualização de comentários, ilustrados na Figura 3, a opção Pesquisar deve estar selecionada, que se opõe a opção Adicionar. Os comentários salvos são acessíveis via toque na tela. Os requisitos para visualização de um comentário em sua íntegra são: o modo pesquisar estar selecionado; a imagem estar no exato nível de zoom em que o comentário foi salvo e; o usuário tocar a imagem em um ponto próximo ao que o comentário foi salvo, com uma tolerância de aproximadamente $10 \%$ visando facilitar a pesquisa. Para a localização do foco, a cada toque na tela, a posição equivalente na imagem é mostrada na parte superior da tela, como ilustrado na Figura 3.
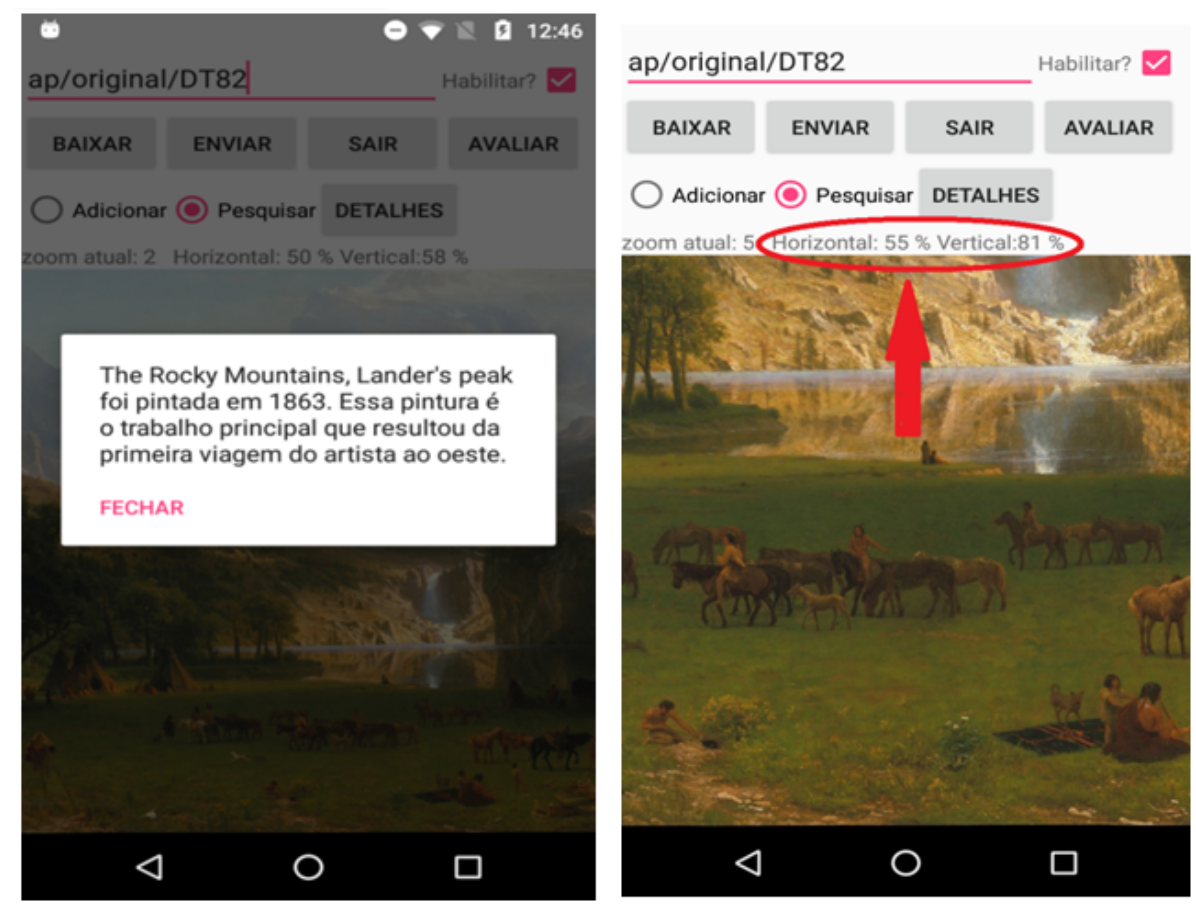

Figura 3. Visualização de comentário e nível de zoom. Obra intitulada "The Rocky Mountains, Lander's Peak"[Bierstadt 1863]

Para auxiliar o usuário na pesquisa por comentários, existe um botão com a opção para a visualização dos detalhes dos comentários. Ao apertar o botão Detalhes são mostrados na tela todos os comentários previamente salvo. Selecionando-se um dos comentários, é possível visualizar a localização do mesmo na imagem. Com a informação da localização, o professor pode voltar a interagir com a imagem, ajustando foco e zoom, para encontrar o comentário e visualizá-lo na íntegra.

Para a avaliação da tarefa foi desenvolvida uma tela que faz a listagem das tarefas 
VI Congresso Brasileiro de Informática na Educação (CBIE 2017)

Anais dos Workshops do VI Congresso Brasileiro de Informática na Educação (WCBIE 2017)

disponíveis para os alunos. Ao selecionar uma tarefa, o professor visualiza os alunos que já a realizaram e enviaram seus comentários.

Após interagir com a tarefa feita pelo aluno, o professor pode encerrar a avaliação informando a situação final para a tarefa, no caso, satisfatório ou não satisfatório.

Para a realização de uma tarefa, por parte do aluno, o aplicativo proporciona a interação dinâmica com zoom, foco e a inserção de comentários e observações. O aluno pode realizar a tarefa na imagem da obra de arte de forma livre ou seguindo e se baseando nos comentários, observações e perguntas do professor.

Após selecionar uma tarefa para ser realizada, o aluno acessará uma tela com a imagem selecionada pelo professor. A tela possui um botão para enviar a tarefa para avaliação, um botão Detalhes para auxiliar a pesquisa de comentários, um botão Sair para voltar a listagem de tarefas, um campo Comentar e um Pesquisar para o modo de interação, um botão para comutar entre comentários do aluno e do professor, um campo com o zoom atual, um campo para o último foco pesquisado e a imagem da obra de arte em destaque na tela.

O aluno, assim como nas funcionalidades para o professor, pode interagir com a imagem alterando, dinamicamente, zoom e foco e inserindo e visualizando comentários. $\mathrm{O}$ aluno faz uso das funcionalidades de zoom e foco para estudar e entender a obra de arte, visualizando detalhes que podem ser imediatamente anotados nos pontos observados. Para inserção de um comentário, o aluno deve manter a seleção no modo Comentar e dar um toque simples no ponto que deseja deixar salvo um registro observado.

O aluno pode visualizar os comentários salvos por ele, bem como os comentários cadastrados pelo professor na criação da tarefa. Com o modo pesquisar selecionado, quando tocado um ponto com comentário salvo na imagem, o comentário aparecerá em uma janela na tela. O botão Detalhes auxilia na localização dos comentários salvos. Com o botão "comentários de:" ligado, os comentários do professor serão visualizados, e com o botão desligado, os comentários do aluno serão visualizados. Com o botão Enviar, a tarefa será enviada e disponibilizada para correção por parte do professor. A persistência das informações geradas durante o uso do aplicativo foi feita usando o Back4app, como descrito anteriormente.

\section{Testes e Resultados}

Os testes foram feitos de forma prática em um ambiente real do problema, sendo, neste caso, turmas do ensino médio da disciplina de artes. O projeto foi submetido e aprovado, por meio da Plataforma Brasil, ao Comitê de Ética da instituição na qual o projeto foi realizado, o qual foi aprovado.

O teste foi realizado com um total de 36 alunos, do primeiro ano do ensino médio da instituição pública. Os testes foram realizados durante as aulas de artes, com acompanhamento da professora responsável pela disciplina.

Foram criadas três tarefas para os alunos que contam com uma obra de arte do Museu Metropolitano de Nova York e alguns comentários com dados históricos da pintura. Os alunos, usando tablets ou smartphones, escolheram uma das tarefas para dar início ao teste. Antes dos alunos iniciarem as tarefas, foram dadas as orientações para o uso das funções disponíveis na aplicação. 
VI Congresso Brasileiro de Informática na Educação (CBIE 2017)

Anais dos Workshops do VI Congresso Brasileiro de Informática na Educação (WCBIE 2017)

As tarefas foram: interagir e se familiarizar com a função de zoom; interagir e se familiarizar com a função de ajuste de foco; encontrar dois ou mais comentários feitos pelo professor; adicionar comentários aos comentários do professor; inserir comentários de forma livre; encontrar os seus próprios comentários e; enviar para avaliação.

O questionário possuia questões abertas, cujo objetivo foi a obtenção dos sentimentos e opiniões dos alunos quanto ao aplicativo e seu uso durante o teste, bem como quanto o uso de tecnologias no ambiente de ensino, e questões fechadas, cujo objetivo era medir o grau de satisfação do aluno com o uso do aplicativo e de tecnologias em sala de aula para apoio ao aprendizado.

A Tabela 1 apresenta os principais resultados em relação às questões abertas. Essas questões tiveram por objetivo apenas a obtenção de uma resposta positiva ou negativa para a sensibilidade do aluno em relação a aplicação, não sendo solicitadas descrições para as dificuldades encontradas.

Tabela 1. Respostas recebidas dos alunos

\begin{tabular}{lll} 
Questão & Sim & Não \\
\hline Encontrou facilidade no uso do aplicativo? & 31 & 5 \\
\hline Conseguiu concluir todas as operações & 35 & 1 \\
\hline Relato de alguma dificuldade? & 20 & 16
\end{tabular}

A Tabela 2 apresenta os resultados das principais questões fechadas. A resposta dessas questões deviam ser respondidas em uma escala de 0 (Discordo Totalmente) a 5 (Concordo Totalmente).

Tabela 2. Respostas recebidas dos alunos

\begin{tabular}{lcccccc} 
Perguntas & Discordo - 1 & 2 & 3 & 4 & Concordo - 5 & Não respondeu \\
\hline Gostaria de utilizar sempre & 0 & 6 & 17 & 2 & 10 & 1 \\
\hline O sistema é complexo & 18 & 6 & 8 & 1 & 1 & 2 \\
\hline O sistema é fácil de usar & 1 & 8 & 9 & 5 & 12 & 1 \\
\hline Preciso de auxílio técnico & 13 & 8 & 7 & 5 & 2 & 1 \\
\hline Sistema confuso & 12 & 9 & 8 & 6 & 0 & 1
\end{tabular}

\section{Conclusões}

Os objetivos específicos foram alcançados com sucesso, pois se chegou a um aplicativo que permite uma interação dinâmica com imagens de obras de arte, além do compartilhamento dos dados e informações gerados entre alunos e professor.

O desenvolvimento para dispositivos móveis pode ser uma porta de entrada para a introdução e contextualização positiva das TIC com os diversos ambientes de ensino. Este trabalho deu um importante passo para se tornar uma ferramenta aplicada de forma contextualizada com o conteúdo desenvolvido em aulas de artes, principalmente pelo fato de ter sido testado no ambiente real do problema, validando hipóteses, buscando melhorias e novas funcionalidades, podendo medir o quão próximo ou distante do ideal o produto desenvolvido está. 
VI Congresso Brasileiro de Informática na Educação (CBIE 2017)

Anais dos Workshops do VI Congresso Brasileiro de Informática na Educação (WCBIE 2017)

Como continuação do projeto, pode-se citar: desenvolvimento de um guia ou tutorial do aplicativo; viabilizar a realização de tarefas em modo offline; permitir modo colaborativo; ampliação do uso para outras disciplinas.

\section{Referências}

Bierstadt, A. (1863). The rocky mountains, lander's peak [pintura]. In Óleo sobre a tela. 73 1/2 $\times 1203 / 4$ in. $(186.7 \times 306.7 \mathrm{~cm})$. Fundo Rogers.

Costa, C. (2013). Educação, imagens e mídias. In Cortez Editora. São Paulo.

David, J. (1787). The death of socrates [pintura]. In Óleo sobre tela, $51 \times 77$ 1/4 pol. $(129,5 \times 196,2 \mathrm{~cm})$. Coleção de Catherine Wolfe Catharine Lorillard, Fundo Wolfe.

Eicher, M. and Pino, J. (2015). Carbópolis. In Carbópolis. Disponível em: http://www.iq.ufrgs.br/aeq/carbopp.htm.

Ferrete, A. and Ferrrete, R. (2015). As tecnologias móveis na formação docente. In Congresso Brasileiro de Informática na Educação, pages 844-853. Universidade Federal de Sergipe.

Lee, W. (2011). Introdução ao desenvolvimento de aplicativos para android. In Editora Ciência Moderna. Rio de Janeiro.

Melo, R. and Carvalho, M. (2014). Aplicativos educacionais livres para mobile learning. In Encontro Virtual de Documentação em Software Livre e Congresso Internacional de Linguagem e Tecnologia Online. XI EVIDOSOL e VIII CILTEC. Disponível em: http://evidosol.textolivre.org.

Mendonça, P. (2015). Materialidade e sociedade: tendências sociotécnicas em tecnologias móveis. In ScientiaeStudia, pages 929-947. São Paulo.

Mercado, L. (2002). Novas tecnologias na educação: reflexões sobre a prática. In Edufal. Campo Grande.

Pedro, L., Borges, S., Lopes, A., Souza, J., Brandao, A., Brandao, L., and Isotani, S. (2012). Projeto e desenvolvimento de um aplicativo de geometria interativa para dispositivos móveis. In Simpósio Brasileiro de Informática na Educação. Rio de Janeiro.

Pretto, N. (2007). Educação, comunicação e a anped: uma história em movimento. In GT: Educação e Comunicação. v. 16.

Ramos, D. (2011). As tecnologias da informação e comunicação na educação: reprodução ou transformação? In ETD - Educação Temática Digital, pages 44-62. Campinas.

Reis, H. (2014). Concepção de um software de geometria interativa utilizando interfaces gestuais para dispositivos móveis. In Dissertação (Mestrado em Ciências da Computação e Matemática) - Curso de Ciências - Ciências da Computação e Matemática Computacional, Instituto de Ciências Matemáticas e de Computação. USP São Carlos.

Rodrigues, R. (2007). Análise e tematização da imagem fotográfica. Brasília.

Silva, M. (2001). Sala de aula interativa: a educação presencial e a distância em sintonia com a era digital e com a cidadania. In Intercom Sociedade Brasileira de Estudos Interdisciplinares da Comunicação XXIV Congresso Brasileiro da Comunicação. Campo Grande. 